\title{
Organization Structure and Operation of Extreme Floods Emergency Management under "Bureaucracy-Cooperation" System in the Huaihe River Basin
}

\author{
Rong $\mathrm{CHEN}^{1, \mathrm{a},{ }^{*} \text {, Hui-Min WANG }}{ }^{1, \mathrm{~b}}$ \\ ${ }^{1}$ State Key Laboratory of Hydrology-Water Resources and Hydraulic Engineering, Hohai University, \\ Nanjing, 210098, China \\ adfcrong@126.com, bhmwang@hhu.edu.cn \\ ${ }^{*}$ Corresponding author
}

Keywords: organization structure, "bureaucracy-cooperation", Huaihe River Basin, extreme floods, emergency management, Stochastic Petri Net

\begin{abstract}
Extreme floods are tending to normalization in the Huaihe River Basin. We proposed that emergency management system of extreme floods in Huaihe River Basin have to integrate bureaucracy with cooperative system, which corresponds with Chinese reality. Therefore, the "bureaucracy-cooperation"(BC) organization structure is constructed. This structure reserves the advantages of high efficiency in bureaucracy and flexibility in cooperative system. And task-team and modular management are emphasized in BC system. Next, we gain further discussion about the $\mathrm{BC}$ organization structure by elaborating its formation mechanism and operation mechanism. Finally, SPN is used to evaluate operational efficiency using data of extreme floods emergency management process in the Huaihe river basin in 2007. The results show that efficiency of an emergency activity, storing floods in a flood detention area, need to be increased yet, and prove that SPN is scientific and applicable to Operational efficiency evaluation.
\end{abstract}

\section{Introduction}

Huaihe River Basin covers some parts of Henan, Anhui, Jiangsu, Shandong and Huber provinces in East China. Located in the North-South climate transition zone, Huaihe River Basin has a wide range of the annual precipitation and uneven distribution of rainfall. These are reasons why Huaihe River Basin is notoriously vulnerable to flooding. In the last decade, two extreme floods have occurred with great losses which are comparable to that in 1954, which is the most severe floods since the founding of PRC.

Frequent occurrence of extreme floods in Huaihe River Basin has caused attention of government. The Huaihe River Commission of the Ministry of Water Resources (HRC) who takes responsibility of water administration of the Huaihe River basin, has accumulated much experience during fighting against floods. And floods emergency plans of Huaihe River Basin have been established and play an indispensable role in fighting floods. Despite this, some management problems still exist, such as barriers between different departments and regions, unclear participation agents, confused distribution of governmental responsibilities, blocked communication, etc. As a result, extreme floods emergency management of Huaihe River Basin is involved in the dilemma of collective action.

In order to get out of the dilemma, we have to improve the current Flood Control and Drought Relief Organization System by static structural support and dynamic action support. On Water Management Forum in 2012, LIU Ning, served as Vice Minister of Ministry of Water Resources, proposed that synergy-management between the normality and the urgency will be a tendency for 
water management. The current management system in the Huaihe River Basin is characterized by bureaucracy, which lacks flexibility in the extreme environment.

In this paper, based on the needs of multi-agent cooperation among central government, HRC and local government, we propose to construct "bureaucracy-cooperation" (BC) organization system that is more in accord with reality and more feasible for extreme floods emergency management in the Huaihe River Basin.

The paper is organized as follows. In the next section, we analyze organizational evolution trend of floods emergency management. In Section 3, BC organization structure is constructed. In Section 4, we present the management mechanism of BC. In Section 5, Stochastic Petri net is used to evaluate operating efficiency. Finally, we conclude our paper in section 6.

\section{Organizational evolution trend of "Bureaucracy-Cooperation"}

Since the founding of PRC, government has put numerous resources into fighting against the floods, and the organizational structure has changed many times. In May 2003, Huaihe River Basin Flood Control and Drought Relief Headquarters (HRFCH) was established by organizing the People's Government of Anhui, Henan, Jiangsu, Shandong Province and HRC. At this point, a bureaucratic flood control system of Huaihe River Basin is basically formed.

Writing in the early 1900s, Max Weber, a German sociologist, described an ideal type of organization which he called a bureaucracy - a form of organization characterized by division of labor, a clearly defined hierarchy, detailed rules and regulations, and impersonal relationships. Many of today's government organizations are designed based on his theory, so does HRFCH.

In the bureaucratic system, National Headquarters, HRC and Local Flood Control Office form hierarchy to carry out water management together. They have definite administrative permissions. There is "principle-agents" between neighboring levels, authority of decision-making in the higher organ and operations in the lower. Then, the higher supervise the lower with executive power. The greatest advantage of this system is highly centralized management like army in fighting against the floods. The top agent commands every emergency activity and dispatches all emergency supplies. This is why we own the best efficient floods emergency power admired by the world.

However, fighting against the floods has been becoming routine to HRC, rather than a usual sense of emergency. Regularity is the real cause of high efficiency. Conversely, the bureaucracy would lose the advantage, even be close to collapse while facing unconventional floods or extreme floods.

Actually, from the 1970s, disadvantages of Bureaucracy have been advert by researchers. In extreme floods emergency management, Bureaucracy is not the best choice. It is manifested as follows: authority of hierarchy is weakened by multi-agent participation, impersonal relationship contradict individual initiative, perfect cooperation is hard to be achieved with professional division of labor, single information pipeline is too narrow to massive information. These problems restrain the efficiency. Therefore, the bureaucratic system in the Huaihe River Basin has to be reformed.

The disadvantages of bureaucracy in emergency management tell us that changing from Bureaucracy to Cooperation becomes inevitable tendency. It should be noted that complete abandoning Bureaucracy is not actual or feasible because of irreplaceable experience of the bureaucracy system in Huaihe River Basin. As the best scheme, BC picks up advantages both of Bureaucracy and Cooperation. In this way, HRFCH continues to handle decision-making and commanding, while other emergency agents should be given appropriate authority of self-decision-making and self-action. 


\section{Organization Structure of "Bureaucracy-Cooperation"}

$\mathrm{BC}$ system is the inevitable trend of extreme floods emergency management in the Huaihe River Basin. "Bureaucracy" here refers to the static organizational system and management mechanisms, while "cooperation" emphasizes the dynamic interaction and response during the emergency process.

In the current organization system, there are dozens of government departments, and the relationships between emergency agents are criss-cross. As previously analyzed, BC system is the best choice to fight against the extreme floods. Some principles should be followed while designing a BC system, such as being people-based and responding scientifically, combining peacetimes with wartimes, being task-oriented and implementing module management and integrating resources and sharing information. Based on the principles, BC system in the Huaihe River Basin is shown in Fig.1.

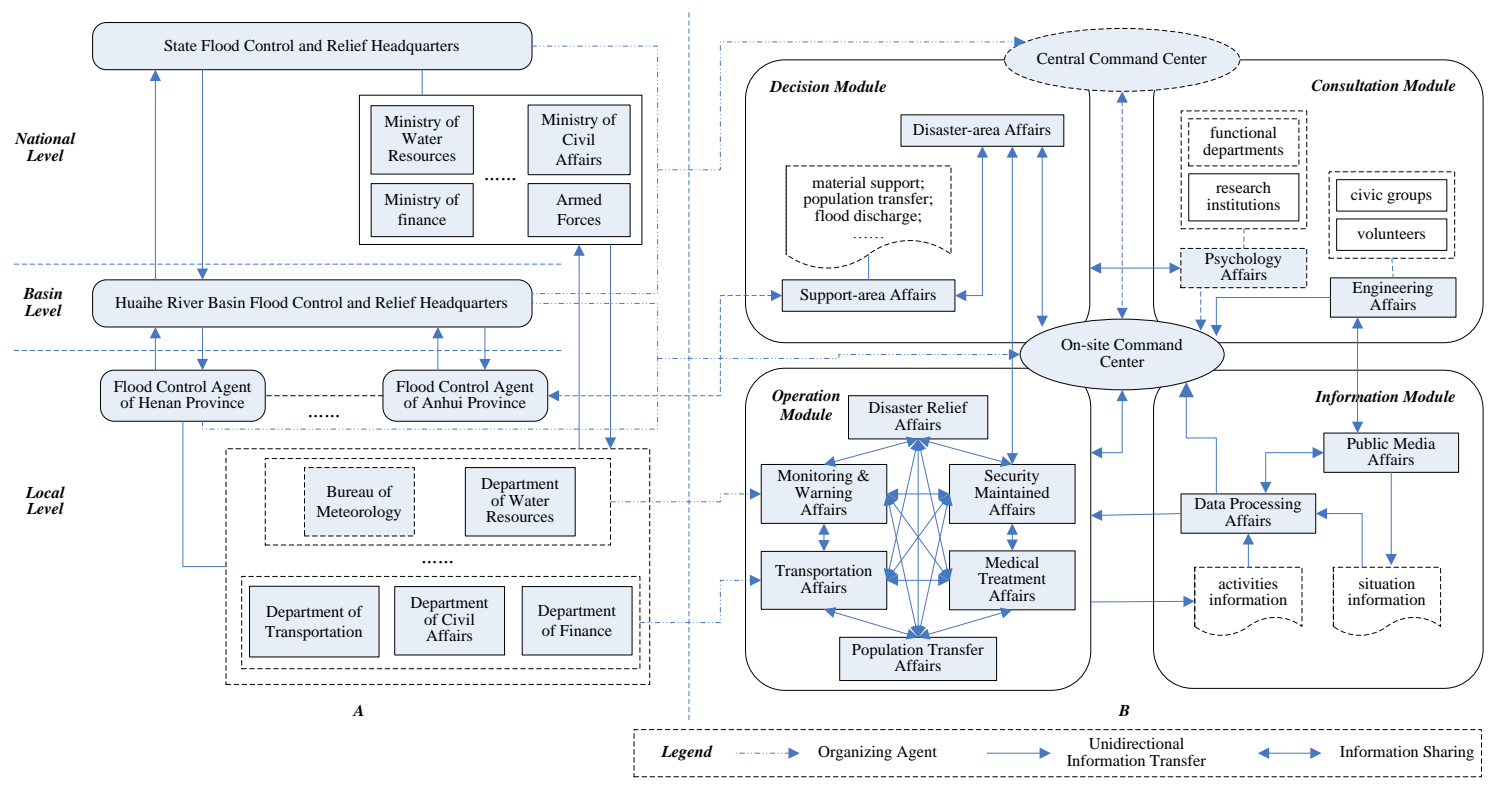

Fig.1 BC Organization Structure of Extreme Floods Emergency Management

Part A, the left of Fig.1, shows the current bureaucratic system in the Huaihe River Basin, which includes national, basin and local levels. And the relevant Headquarters are set up to be responsible for making emergency plans, commanding and coordinating with the lower, supervising the implementation of the emergency plans and making adjustments. The government functional departments in the system are entitled to the necessary human, material, financial and other resources. And command is conveyed from top to down while information is reflected form bottom to up.

Facing invasion of extreme floods, the organization structure for fighting against extreme floods in the Huaihe River Basin should be changed into BC system, shown as part A plus part B in Fig.1. We borrow CMSS[1](Crisis management shell structure) mode, a cooperation structure of modern emergency management, to design the $\mathrm{BC}$ organizational structure. CMSS mode is a relatively full-featured system designed for large organizations to deal with the crisis. It mainly consists of four functional modules, namely decision-making module, consultation module, information module and operation module that are of equal importance, as shown in Part B of Fig.1.

Part B, the right of Fig.1, is about the task functions in the cooperation between the basin and the region, as well as the form of information transfer. In practice, the four modules, including 
decision-making, consulting, operations and information, act at regional level while HRC continues to play command and coordination functions through command center.

(1) Command Centers. Considering classification management, we set up 2-level command centers, namely the central command center and on-site command center, both belonging to the decision-making level. They continue the unified command, which will be able to quickly allocate kinds of resources and efficiently deal with floods.

(2) Modular Management. In modules of decision-making, consulting, operations and information, task teams and modular management give a full performance of cooperation's flexibility, which ensures effective communication between emergency agents and makes up shortage of the bureaucracy.

\section{Management Mechanism of "Bureaucracy-Cooperation"}

\section{The formation mechanism of "bureaucracy-cooperation"}

The formation mechanism of BC system is showed in Fig.2.

Bureaucracy is applicable to fighting against the regular floods with high-efficiency. It is indecisive when crisis is coming. At this moment, the staffs in the bureaucratic agent become non-adapted and anxious. As long as their emotions get a threshold quantity, the bureaucratic organization system will lose advantage of high-efficiency, even sink into chaos.

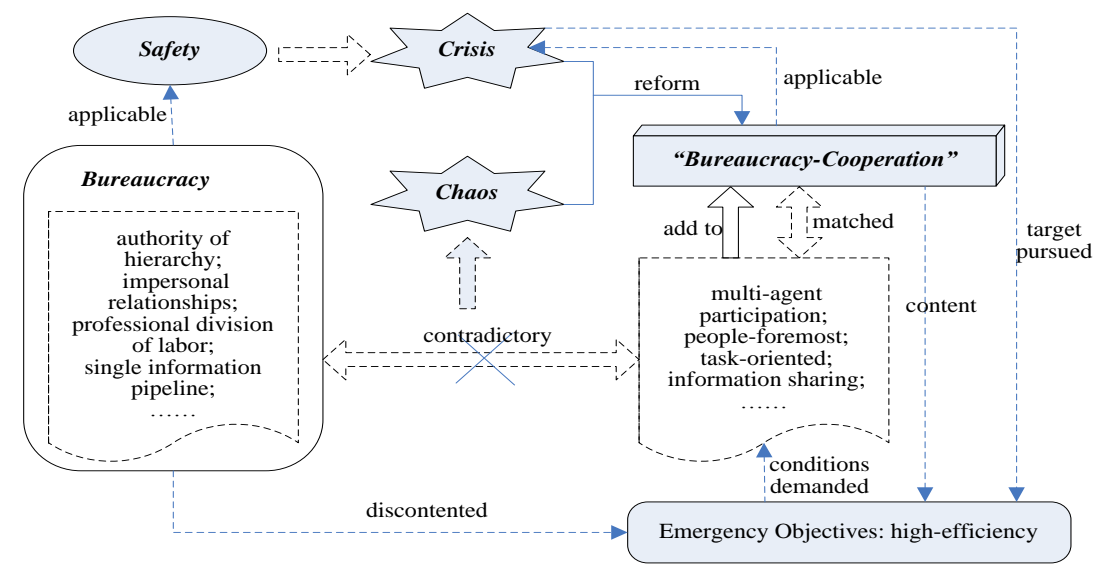

Fig.2 The Formation Mechanism of BC System

In chaos, individuals in the bureaucratic system begin to adjust their behavior patterns whose individual initiative is aroused. They get out of the hierarchical cage, and contact with each other based on some relationships about neighborhood, kinship, profession, etc, and organize task teams within task-oriented and mutual trust. As a result, cooperation initiates from the hierarchical organization system, and $\mathrm{BC}$ is formed.

\section{The operation mechanism of "bureaucracy-cooperation"}

The operation mechanism of BC system is showed in Fig.3. 


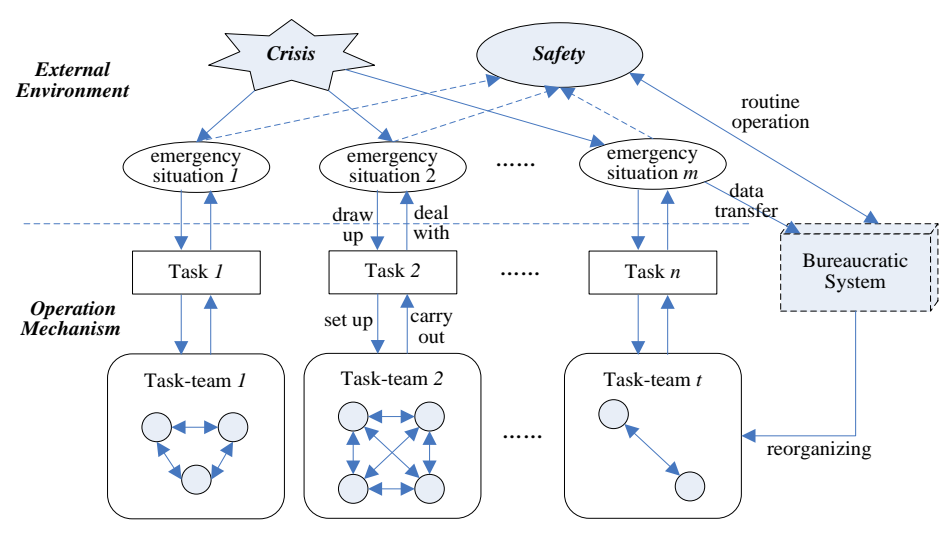

Fig.3 The Operation Mechanism of BC System

Firstly, the data about crisis transfer to regularly bureaucracy system. Secondly, individuals working in reorganize groups with some special relationships, called "node". For example, we need "doctor-node" for medical treatments in extreme floods emergency management, likewise "nurse-node". Thirdly, a node connects with other nodes and carries out the task formed with a task team. In a task team, the number of nodes is positively correlated with complexity of the task.

In fact, emergency situations occur simultaneously in a flood disaster. A node participates in more than one task team simultaneously, while information and resources shared among task teams. Then, a huge network architecture is formed by a large quantity of nodes. In this network, the speed of information dissemination increases exponentially, which makes less blockage and distortion and higher efficiency of emergency management.

\section{Operating efficiency evaluation of "Bureaucracy-Cooperation"}

Extreme floods emergency management is a process racing against time. The more timely floods is controlled, the less disaster losses. Therefore, the input is often difficult to be considered rationally and be quantified in emergency response process. So, general efficiency evaluation methods are not applicable. We need an evaluation method with less demanding on quantitative indicators. Stochastic Petri net (SPN) is the best choice because of characteristic of visualization and powerful mathematical support. SPN can intuitively describe the relationship of activities, and analyze the static structure by means of mathematical methods, as well as the dynamic action performance.

A Stochastic Petri Net (SPN) model is a 6-tuple[4], $S P N=\left(\mathrm{P}, T, F, W, M_{0}, \lambda\right)$, where $P=\left\{p_{1}, p_{2}, \cdots, p_{n}\right\}$ is a finite set of places, $T=\left\{t_{1}, t_{2}, \cdots, t_{m}\right\}$ is a finite set of transitions, and $F \subseteq(P \times T) \cup(T \times P)$ is a set of arcs where an arc connects a transition to place or a place to a transition. $W: F \rightarrow\{1,2, \cdots\}$ is a set of weight functions, $M_{0}: P \rightarrow\{1,2, \cdots\}$ is the initial marking, and $\lambda=\left\{\lambda_{1}, \lambda_{2}, \cdots, \lambda_{m}\right\}$ is a set of average firing rates of transitions.

Considering time limit and different key emergency missions, the whole process is divided into three phases: disaster forecasting, emergency response and after-flood reconstruction. The key 
emergency mission in forecasting phase is monitoring and early warning, and summarizing in after-flood phase.

The key mission in response phase is fighting against floods and reducing disaster losses. This phase is divided into three sections. Section 1: The flood just begins to take shape, and the core task is that project management units execute flood scheduling program by permission. Section 2: The water level of the chief hydrological stations is closing to the warning water level and continues increasing. The core task is organizing consultations to discuss strategy. Flood detentions are usually to be considered to use or not. Section 3: Dam might be broken for long-term immersion, and the core task is strengthening projects' guard and repair.

Following this phase and sectional situations, we design the SPN model as shown in Fig.4. In the SPN model, the transitions $t_{m}$ are used to represent the emergency response activities, and the places $p_{n}$ represent the emergency processing situations.

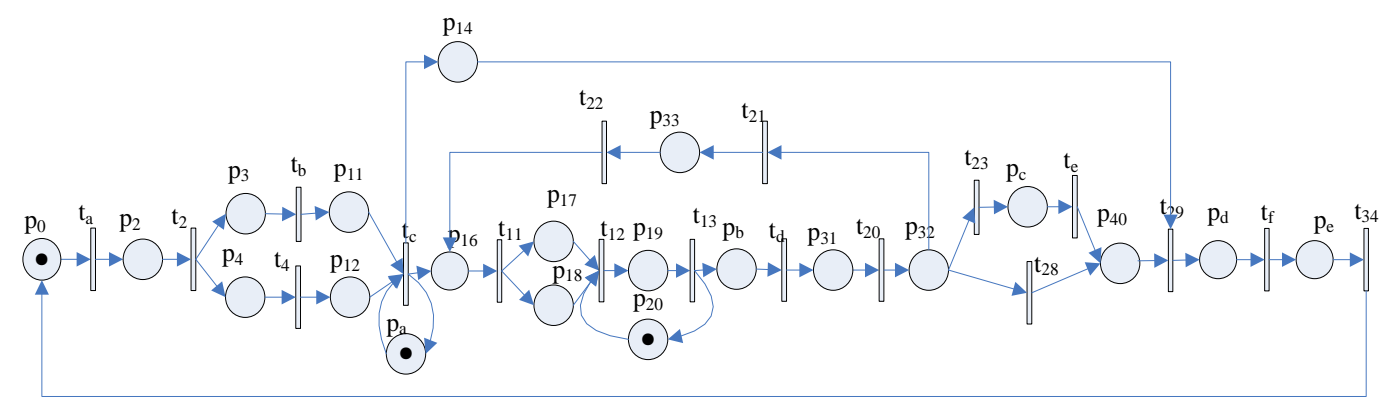

Fig.4 The SPN model of extreme floods emergency management

To evaluate the performance of the SPN model, we usually use a Markov Chain (MC) which has the same performance with the SPN model. For the MC, we can obtain the steady-state probability based on the following $[5,6]$ :

$$
\left\{\begin{array}{c}
P Q=0 \\
\sum_{i=0}^{k} P\left(M_{i}\right)=1
\end{array}\right.
$$

where $P=\left(P\left(M_{0}\right), P\left(M_{1}\right), \cdots, P\left(M_{k}\right)\right)$ represents a row vector of the probability distribution of steady state. $Q=\left[q_{i j}\right],(0 \leq i, j \leq k)$ represents a state transition matrix. $q_{i j}$ is defined as:

$$
q_{i j}=\left\{\begin{array}{c}
\lambda_{i j}, i \neq j \text { and there exists an arc from } i \text { to } j \\
-\sum_{j=0}^{k} \lambda_{i j}, \text { there are } k \text { arcs depart from } i .
\end{array}\right.
$$

The busy probability of places indicator can be calculated by (3). The utilization of transitions indicator can be calculated by (4). 


$$
\begin{aligned}
& P[M(p)=i]=\sum_{j} P\left[M_{j}\right], M_{j} \in\left[M_{0}\right\rangle, M_{j}(p)=i \\
& U(t)=\sum_{M \in E} P[M]
\end{aligned}
$$

To validate the SPN model, we applied the process to extreme floods in the Huaihe River Basin in 2007. The data come from almanac of water conservancy by sorting out and calculating. A set of average firing rates of transitions is given:

$$
\lambda=\{0.96,50,0.4667,1,0.3468,0.4285,0.7142,50,0.2314,0.5286,12,12,50,0.9333,0.25,1,0.0333,1\}
$$

. Then calculate the busy probability of places and the utilization of transitions by (3) and (4).

The calculation results of the busy probability of places show that places represent information feedback are almost 1 . The ones represent conventional reservoir operation and the state of flood detentions are also higher than others. We need to focus on these places by improving plan system ahead of schedule. The calculation results of the utilizations of transitions show that transition represents summary after disaster and the one represent population transfer are relatively higher. The latter is time-pursuing and a miniature of cooperation between basin government and local government. By optimizing this miniature, we could put the principle of "cooperation" included in $\mathrm{BC}$ into practice.

\section{Conclusions}

In this paper, we proposed a new organizational evolution tendency that is from Bureaucracy to BC. Bureaucratic system is applicable to the regular floods with high-efficiency. But when crisis is coming, cooperation is more needed. However, it's not actual or feasible to completely abandon Bureaucracy. As the best scheme, BC picks up advantages both of Bureaucracy and Cooperation. Based on this, we construct organization structure combining characteristics of bureaucracy and cooperation with 2-level command centers, task teams and modular management. We analyze formation mechanism and operation mechanism. And we proposed a method to evaluate the floods response process based on SPN. The case emulation using data of flood disaster in the Huaihe River Basin in 2007 proves that the method is useful to find the problems in the emergency response process and provided some suggestions for improvement.

\section{Acknowledgements}

The research work was supported by National Natural Science Foundation of China under Grant No. 90924027 and National Social Science Foundation of China under Grant No. 12\&ZD214.

\section{References}

[1] Heath R. Dealing with the Complete Crisis_-the Crisis Management Shell Structure[J]. Safety Science. 1998, 30: 139-150. 
[2] Bigley G A, Roberts K H. The Incident Command System: High-Reliability Organizing for Complex and Volatile Task Environments[J]. The Academy of Management Journal. 2001, 44(6): 1281-1299.

[3] Kim-Farley R J, Celentano J T, Gunter C, et al. Standardized Emergency Management System and Response to a Smallpox Emergency[J]. Prehosp Disaster Med. 2003, 18(4): 313-320.

[4] Shan S, Wang L, Li L. Modeling of emergency response decision-making process using stochastic petri net: an e-service perspective[J]. Information Technology and Management. 2012(13): 363-376.

[5] Li D, Sun X, Gao J, et al. Reachability determination in acyclic Petri nets by cell enumeration approach[J]. Automatica. 2011, 47(9): 2094-2098.

[6] German R. Markov regenerative stochastic petri nets with general execution policies: supplementary variable analysis and a prototype tool[J]. Performance Evaluation. 2000, 39: 165-188. 